\title{
Properties of Quasar and AGN Candidates Detected in the 2MASS/ROSAT Catalogs
}

\author{
Shinjirou Kouzuma ${ }^{1}$ and Hitoshi Yamaoka ${ }^{1}$ \\ ${ }^{1}$ Graduate School of Sciences, Kyushu University, Fukuoka 812-8581, Japan \\ Email: kouzuma@phys.kyushu-u.ac.jp
}

Keywords. galaxies: active, quasars: general, catalogs

Color selection is an efficient technique for extracting candidate AGNs. Many AGNs have been selected on the basis of colors at various wavelengths. In this work, we extracted AGN candidates using near-infrared colors and investigated their properties.

We first cross-identified both the catalog of quasars and AGN of Véron-Cetty \& Véron (2006) and SDSS-DR5 quasar catalog III with the 2MASS point source catalog (Cutri et al. 2003), and confirmed quasar/AGN loci in a near-infrared $\left(H-K_{\mathrm{S}}\right) /(J-H)$ color-color diagram as the loci of AGNs are different from the stellar loci. We propose the following near-infrared color selection criteria for extracting AGN candidates: $(J-H) \leqslant 1.70\left(H-K_{\mathrm{S}}\right)-0.118$ and $(J-H) \leqslant 0.61\left(H-K_{\mathrm{S}}\right)+0.50$.

We extracted bright sources at both near-infrared and X-ray wavelengths by positional crossidentification between 2MASS and ROSAT, using both the Bright Source Catalogue (BSC; Voges et al. 1999) and the Faint Source Catalogue (FSC; Voges et al. 2000). We set the positional criterion to be $\leqslant 30^{\prime \prime}$. AGN candidates were selected from the cross-identified sources using the near-infrared color selection criteria described above, resulting in $\sim 5,000(10,000)$ candidates from the BSC (FSC) catalog.

To check the possibility that the AGN locus in the near-infrared color-color diagram is contaminated by other types of objects, we examined loci of four types of objects that emit non-thermal radiation or are considered to be bright at both near-infrared and X-ray wavelengths: microquasars, cataclysmic variables (CVs), low-mass X-ray binaries (LMXBs), and massive young stellar objects (MYSOs). In each case, few objects are located around the AGN locus in the near-infrared color-color diagram so these objects are unlikely to contaminate the AGN locus. In addition, we examined the near-infrared colors of quasars by performing a Monte Carlo simulation using the HYPERZ code. Simulated colors are consistent with the colors of cataloged quasars/AGNs.

Multiwavelength counterparts were extracted by cross-identification with several catalogs (i.e., USNO-B1.0, SDSS, MSX, and IRAS). In particular, we investigated optical properties on a $\left(u^{\prime}-g^{\prime}\right) /\left(g^{\prime}-r^{\prime}\right)$ color-color diagram using SDSS photometry. The optical colors of AGN candidates are clearly different from stellar colors and are similar to those of SDSS quasars. Therefore, we conclude that we can select AGN candidates on the basis of near-infrared colors, and it is highly probable that our candidates are AGNs.

\section{References}

Cutri, R. M., et al. 2003, 2MASS All Sky Catalog of Point Sources, NASA/IPAC Infrared Science Archive (Pasadena: Caltech)

Véron-Cetty, M. P. \& Véron, P. 2006, A\&BA, 455, 773

Voges, W., et al. 1999, A\&̈A, 349, 389

Voges, W., et al. 2000, IAU Circ. No. 7432, 3 